\title{
Concurrent resistance and aquatic treadmill training elicits greater lean mass gains than resistance training alone.
}

Brad S. Lambert (Doctoral Student), Nicholas P. Greene, John S. Green (FACSM), Stephen F. Crouse (FACSM)

PURPOSE: To examine the effects of combined aquatic treadmill training (ATM) and resistance training (RT) on body composition (Bcomp), strength, and $\mathrm{VO}_{2 \max }$ following $12 \mathrm{wks}$ of training compared to RT alone. METHODS: 5 healthy, sedentary men $(32.6 \mathrm{yr} \pm 11,187.5 \mathrm{~cm} \pm 4.9,107.9 \mathrm{~kg} \pm 19.5)$ and 6 women (34.2 yrs $\pm 14.3,166.6 \mathrm{~cm} \pm 8.4,78.6 \mathrm{~kg} \pm 19.69$ ) were recruited and screened to assess $\mathrm{VO}_{2 \max }(\mathrm{GXT})$, Bcomp (DEXA), and strength. During strength assessment, 1RMs were assessed in this order: leg press, chest press, leg curl, lat pull, leg ext, triceps push-down, biceps curl. Subjects were then randomized into 2 groups. Both groups $(\mathbf{R}=$ resistance training, $\mathbf{C T}=$ concurrent training $)$ performed progressive $\mathrm{RT}$ for 12 wks ( $2 /$ wk, 3 x 8-12 @ 60\%1RM, 1.5min rest int.). Following wk 6, 1RMs were reassessed and intensity was increased for wks 7-12 (3 x 4-8, beginning @ 75\%1RM). Concurrently, the CT group performed 12 wks of progressive ATM $\left(60 \rightarrow 85 \% \mathrm{VO}_{2 \max }\right)$. $\mathrm{VO}_{2 \max }$ was reassessed following wk 6 . ATM occurred immediately following RT sessions and in isolation on a $3^{\text {rd }}$ day during the wk. After training, baseline tests were again performed. A $2 \times 3$ factorial ANOVA w/ repeated measures for group (2) by time (3) was used to examine absolute and relative changes in body composition, strength, and $\mathrm{VO}_{2 \max }$.

\section{RESULTS: (TABLE)}

\begin{tabular}{|c|c|c|c|c|c|c|}
\hline Independent Var. & Group & Baseline & Wk 6 & Wk 12 & $\% \Delta$ Wk 6 & $\% \Delta \mathrm{Wk} 12$ \\
\hline \multirow{2}{*}{$\mathrm{VO}_{2 \max }(\mathrm{L} / \mathrm{min})$} & $\mathrm{CT}^{\mathrm{m}=3 \mathrm{f}=3}$ & $3.1 \pm 0.36^{\mathrm{a}}$ & $3.18 \pm 0.34$ & $3.37 \pm 0.37^{\mathrm{a}}$ & $3.35 \% \pm 2.53^{b}$ & $9.2 \% \pm 3.13^{\mathrm{a}, \mathrm{b}, \dagger}$ \\
\hline & $R^{m=2 \mathrm{f}=3}$ & $2.66 \pm 0.39$ & $2.72 \pm 0.39$ & $2.74 \pm 0.35$ & $3.11 \% \pm 3.84$ & $4.65 \% \pm 5.28^{\dagger}$ \\
\hline \multirow{2}{*}{ Fat Mass (kg) } & $\mathrm{CT}$ & $34.86 \pm 4.48^{a}$ & & $\pm 4.46^{\mathrm{a}}$ & $-1.15 \% \pm 1.26$ & $-3.93 \% \pm 1.57^{\mathrm{a}, \dagger}$ \\
\hline & $\mathrm{R}$ & $34.04 \pm 7.49$ & $53.54 \pm 1.45$ & $34.08 \pm 7.29$ & $-1.65 \% \pm 0.34$ & $0.68 \% \pm 1.02^{\dagger}$ \\
\hline \multirow{2}{*}{ Lean Mass (kg) } & CT & $55.49 \pm 6.35^{a}$ & $57.01 \pm 6.61^{b}$ & $58.98 \pm 6.9^{\mathrm{a}, \mathrm{b}, \uparrow}$ & $2.63 \% \pm 0.75^{b}$ & $6.11 \% \pm 1.13^{\mathrm{a}, \mathrm{b}, \dagger}$ \\
\hline & $\mathrm{R}$ & $49.51 \pm 6.48^{\mathrm{a}}$ & $51.16 \pm 6.57^{\mathrm{a}}$ & $50.28 \pm 6.49^{\dagger}$ & $3.46 \% \pm 0.69^{\mathrm{a}}$ & $1.63 \% \pm 1.29^{\dagger}$ \\
\hline \multirow{2}{*}{ Leg Press (lbs) } & CT & $752.1 \pm 112.3^{\mathrm{a}}$ & $878.5 \pm 157.9^{b}$ & $1072.1 \pm 187.2^{\mathrm{a}, \mathrm{b}}$ & $14.68 \% \pm 4.66^{\mathrm{a}, \mathrm{b}}$ & $41.19 \% \pm 7.79^{\mathrm{a}, \mathrm{b}}$ \\
\hline & $\mathrm{R}$ & $650.3 \pm 92.4^{\mathrm{a}}$ & $754 \pm 91.8$ & $9.6 \pm 129.1^{\mathrm{a}}$ & $17.58 \% \pm 3.20^{\mathrm{a}, \mathrm{b}}$ & $35.59 \% \pm 3.14^{\mathrm{a}, \mathrm{b}}$ \\
\hline \multirow{2}{*}{ Chest Press (lbs) } & CT & $109.6 \pm 21.7^{\mathrm{a}}$ & $130.5 \pm 25.7^{\mathrm{a}, \mathrm{b}}$ & $54.3 \pm 30.7^{\mathrm{a}, \mathrm{b}}$ & $19.47 \% \pm 3.41^{\mathrm{a}, \mathrm{b}}$ & $41.03 \% \pm 6.4^{\mathrm{a}, \mathrm{b}}$ \\
\hline & $\mathrm{R}$ & $93.4 \pm 17.7^{\mathrm{a}}$ & $111.6 \pm 21.7$ & $117.8 \pm 20.6^{a}$ & $19.6 \% \pm 3.54^{\mathrm{a}}$ & $28.27 \% \pm 4.17^{\mathrm{a}}$ \\
\hline \multirow{2}{*}{ Sum all Lifts (lbs) } & $\mathrm{CT}$ & $1608.5 \pm 223.5^{\mathrm{a}}$ & $1915.5 \pm 297.8^{\mathrm{a}, \mathrm{b}}$ & $2282.1 \pm 329.2^{a, b}$ & $18.09 \% \pm 3.6^{\mathrm{a}, \mathrm{b}}$ & $40.11 \% \pm 5.17^{\mathrm{a}, \mathrm{b}}$ \\
\hline & $\mathrm{R}$ & $1381.1 \pm 174.8^{\mathrm{a}}$ & $1651.8 \pm 234.8$ & $1873 \pm 278.6^{a}$ & $19.06 \% \pm 3.19^{\mathrm{a}, \mathrm{b}}$ & $34.89 \% \pm 5.22^{\mathrm{a}, \mathrm{b}}$ \\
\hline
\end{tabular}

Values are means \pm SE. $\% \Delta=\%$ Change from baseline. Like letters = sig. diff. $w /$ in groups across time, $\dagger=$ diff. between groups for change $(\Delta)$ and $\% \Delta$ at the same time point (comparisonwise $\alpha=0.05$ ).

CONCLUSION: Concurrent RT and ATM elicits greater increases in lean mass than RT alone with the added benefits of improved cardiovascular capacity and reduced fat mass. Partial support from the Huffiness Institute for Sports Medicine and Human Performance. 